\title{
Experiencia clínica con el uso ambulatorio de tolvaptan. Costes y efectividad en nueve casos
}

David E. Barajas-Galindo, ${ }^{1 *}$ Alfonso Vidal-Casariego, ${ }^{1}$ Emilia Gómez-Hoyos ${ }^{2}$ y María Guerra-González ${ }^{3}$

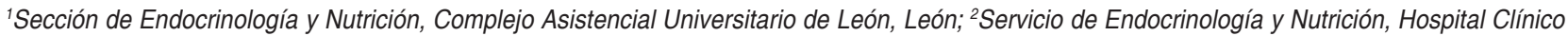
Universitario, Valladolid; ${ }^{3}$ Servicio de Farmacia Hospitalaria, Complejo Asistencial Universitario de León, León. España

\section{Resumen}

Introducción: La introducción de tolvaptan ha supuesto la principal novedad en el tratamiento de la hiponatremia en los últimos años. Objetivo: Describir la experiencia con tolvaptan en el Complejo Asistencial Universitario de León, España. Método: Estudio observacional retrospectivo de utilización ambulatoria de tolvaptan en un hospital de tercer nivel, de marzo de 2014 a agosto de 2017. Resultados: Fueron tratados con tolvaptan de forma ambulatoria nueve pacientes, $23.1 \%$ alcanzó eunatremia en 24 horas. Posterior a la administración de tolvaptan se registró reducción en días de hospitalización (361 versus $70, p=0.007$ ), especialmente por hiponatremia (306 versus $49, p=0.009$ ). Conclusiones: El uso a largo plazo de tolvaptan parece ser seguro y se relaciona con descenso en los días de hospitalización.

PALABRAS CLAVE: Tolvaptan. Hiponatremia. Sodio. Secreción inadecuada de hormona antidiurética.

\section{Clinical experience with Tolvaptan outpatient use. Cost and effectiveness in 9 cases}

\begin{abstract}
Introduction: Tolvaptan introduction has constituted the main therapeutic novelty in the management of hyponatremia in recent years. Objective: To describe the experience with this drug at Complejo Asistencial Universitario de León, Spain. Method: Retrospective, observational study of tolvaptan outpatient use in a tertiary care hospital from March 2014 to August 2017. Results: $A$ total of 9 patients were treated with tolvaptan in the outpatient setting. Eunatremia was reached in $24 \mathrm{~h}$ by 23.1\%. After tolvaptan administration, a reduction in days of hospitalization was recorded (361 vs. $70 ; p=0.007$ ), especially in those days of hospitalization that were attributable to hyponatremia (306 vs. 49; $p=0.009$ ). Conclusions: Long-term use of tolvaptan appears to be safe and is associated with a decrease in days of hospitalization.
\end{abstract}

KEY WORDS: Tolvaptan. Hyponatremia. Sodium. Syndrome of inappropriate antidiuretic hormone secretion.

\section{Introducción}

La hiponatremia, descenso de concentración sérica de sodio por debajo de $135 \mathrm{mmol} / \mathrm{L}$, es el trastorno electrolítico más prevalente en el medio ambulatorio y hospitalario; ${ }^{1}$ su primera causa es la secreción inadecuada de hormona antidiurética, producida fisiopatológicamente por incapacidad para suprimir la secreción de vasopresina. ${ }^{2}$

La hiponatremia leve o moderada $(\mathrm{SNa}=120$ $135 \mathrm{mmol} / \mathrm{L})$, que en muchos casos se mantiene de forma crónica, se asocia con incremento de la mortalidad hospitalaria ${ }^{3}$ y ambulatoria, ${ }^{4}$ así como con aumento de la inestabilidad, caídas y fracturas. ${ }^{5}$ La necesidad de abordar su tratamiento se ha puesto de
Correspondencia:

${ }^{*}$ David E. Barajas-Galindo

E-mail: dabarajas@saludcastillayleon.es
Fecha de recepción: 30-04-2019

Fecha de aceptación: 30-05-2019

DOI: 10.24875/GMM.19005250
Gac Med Mex. 2020;156:78-81

Disponible en PubMed

www.gacetamedicademexico.com CC BY-NC-ND (http://creativecommons.org/licenses/by-nc-nd/4.0/). 
manifiesto en diversos documentos de consenso y guías de práctica clínica, ${ }^{6}$ que proponen la restricción hídrica o el incremento de acuaresis con diuréticos de asa, urea o antagonistas de los receptores V2 de vasopresina, que constituyen la principal novedad terapéutica en su manejo; en Europa se dispone de tolvaptan (TV). ${ }^{7}$

\section{Aportación a la literatura científica}

El propósito del presente estudio fue valorar costes y efectividad del uso ambulatorio de tolvaptan en el Complejo Asistencial Universitario de León, España, entre marzo de 2014 y agosto de 2017, con la finalidad de aportar conocimiento sobre el uso de TV.

Se planteó la hipótesis de que los episodios de hospitalización y visitas a urgencias disminuyen con el uso de TV respecto al periodo sin tratamiento con TV. El coste elevado de este fármaco y el hecho de constituir la principal novedad en el tratamiento de la hiponatremia en los últimos años motivaron el presente reporte.

\section{Método}

Estudio observacional retrospectivo de la utilización de TV de forma ambulatoria en un hospital de tercer nivel. Se incluyeron todos los pacientes mayores de 18 años tratados con tolvaptan entre marzo de 2014 y agosto de 2017; la recolección de datos se realizó de la historia clínica del paciente. La información fue manejada de forma anónima.

Las variables estudiadas fueron edad, sexo, motivo de ingreso o de consulta en urgencias, episodios de hospitalización y urgencias antes y después del tratamiento ambulatorio, causa de hiponatremia, dosis de inicio y mantenimiento, SNa de inicio, así como la última medición disponible, duración del tratamiento y costes directos relacionados con el tratamiento, calculados en función del precio de venta del laboratorio, las dosis dispensadas y el número de días del tratamiento.

Se realizó análisis estadístico con Stata versión 14. Las variables sin distribución normal se describieron como medianas y rangos intercuartílicos y se compararon mediante $\mathrm{U}$ de Mann-Whitney o prueba de Kruskal-Wallis. Las variables categóricas se resumieron como porcentajes y se compararon mediante $\chi^{2}$. Para todas las pruebas se consideró significación estadística con $p<0.05$.

Este estudio se llevó a cabo de acuerdo con las directrices establecidas en la Declaración de
Fortaleza y todos los procedimientos realizados en pacientes humanos fueron aprobados por el Comité de Ética y de Investigación Clínica del hospital. Al ser un estudio retrospectivo y dada la dificultad de obtener el consentimiento informado de cada paciente, este no fue aplicado.

\section{Resultados}

En total, nueve pacientes recibieron TV de forma ambulatoria; sus características se resumen en la Tabla 1.

La dosis de mantenimiento fue inferior a la indicada en la ficha técnica ( $15 \mathrm{mg} / \mathrm{día}$ ) en siete pacientes, consiguiendo eunatremia con $2.1 \mathrm{mg} / \mathrm{día}$ ( $15 \mathrm{mg} / \mathrm{siete}$ días) en $11.1 \%$, con $3.75 \mathrm{mg} / \mathrm{día}$ ( $7.5 \mathrm{mg} / 48$ horas) en $22.2 \%$ y con $7.5 \mathrm{mg} / \mathrm{día}$ (7.5 mg/24 horas) en $55.6 \%$.

No se registraron episodios de hipernatremia ( $\mathrm{Na}>145 \mathrm{mmol} / \mathrm{L}$ ) ni insuficiencia renal aguda durante el seguimiento.

Al comparar en cada paciente los periodos de tratamiento ambulatorio con TV respecto a periodos de tiempo equivalentes previos al inicio de este, encontramos reducción en los días totales de hospitalización (361 versus $70, p=0.007$ ) a expensas de las hospitalizaciones por hiponatremia (306 versus 49, $p=0.009$ ), con tendencia a la reducción en el número de episodios totales de hospitalización (19 versus 7 , $p=0.072)$ y especialmente en los atribuibles a hiponatremia (14 versus $4, p=0.051$ ), así como en episodios de asistencia en urgencias por cualquier causa (18 versus $11, p=0.302$ ) y en los atribuibles directamente a hiponatremia ( 15 versus $5, p=0,076$ ).

El coste total de los tratamientos ambulatorios durante el periodo analizado fue de $170875.7 €$ y la media del gasto por paciente fue de $17930.6 €$ (3733.7-30 766.1).

En la Tabla 2 se desglosa el gasto por diagnóstico y se relaciona el coste y la diferencia registrada en los eventos valorados: episodios de ingreso, días de ingreso y episodios de urgencias.

\section{Discusión}

En los pacientes analizados, la utilización ambulatoria de TV demostró ser segura y eficaz en la reducción de eventos clínicos, incluso con dosis inferiores a las indicadas en la ficha técnica. Por el estudio SALTWATER $^{8}$ disponemos de los datos de seguridad y eficacia del uso crónico TV sobre la evolución de la natremia, sin embargo, la experiencia es limitada en cuanto a los 
Tabla 1. Características de los pacientes que reciben tolvaptan de forma ambulación, en función de la etiología de la hiponatremia

\begin{tabular}{|c|c|c|c|c|c|c|c|c|c|c|c|c|}
\hline Variable & \multicolumn{2}{|c|}{$\begin{array}{c}\text { SIADH } \\
\text { farmacológica } \\
n=3 \\
(33.3 \%)\end{array}$} & \multicolumn{2}{|c|}{$\begin{array}{c}\text { SIADH } \\
\text { paraneoplásica } \\
n=2 \\
(22.2 \%)\end{array}$} & \multicolumn{2}{|c|}{$\begin{array}{c}\text { SIADH } \\
2^{\circ} \\
\text { padecimiento } \\
\text { neurológico } \\
n=2 \\
(22.2 \%)\end{array}$} & \multicolumn{2}{|c|}{$\begin{array}{c}\text { SIADH } \\
2^{\circ} \\
\text { padecimiento } \\
\text { respiratorio } \\
n=1 \\
(11.1 \%)\end{array}$} & \multicolumn{2}{|c|}{$\begin{array}{c}\text { Hiponatremia } \\
\text { dilucional } \\
\text { (cirrosis) } \\
n=1 \\
(11.1 \%)\end{array}$} & \multicolumn{2}{|c|}{$\begin{array}{l}\text { Total } \\
n=9 \\
(100)\end{array}$} \\
\hline \multirow[t]{2}{*}{ Hombre/mujer (\%) } & \multicolumn{2}{|c|}{$33.3 / 66.6$} & \multicolumn{2}{|c|}{$100 / 0$} & \multicolumn{2}{|c|}{$0 / 100$} & \multicolumn{2}{|c|}{$100 / 0$} & \multicolumn{2}{|c|}{$100 / 0$} & \multicolumn{2}{|c|}{$55.6 / 44.4$} \\
\hline & Me & DQ1-Q3 & Me & DQ1-Q3 & $\mathrm{Me}$ & DQ1-Q3 & Me & DQ1-Q3 & Me & DQ1-Q3 & Me & DQ1-Q3 \\
\hline Edad (años) & 79.1 & 17.1 & 70.2 & 20.23 & 67.8 & 6.2 & 86.4 & 0 & 56.9 & 0 & 70.9 & 15.6 \\
\hline SNa inicio (mmol/L) & 123 & 5 & 121 & 8 & 125.5 & 7 & 131 & 0 & 113 & 0 & 123 & 6 \\
\hline SNa final $(\mathrm{mmol} / \mathrm{L})$ & 139 & 5 & 138.5 & 3 & 138.5 & 5 & 142 & 0 & 124 & 0 & 139 & 4 \\
\hline Dosis inicio TV (mg/día) & 7.5 & 0 & 11.25 & 7.5 & 11.25 & 7.5 & 7.5 & 0 & 7.5 & 0 & 7.5 & 0 \\
\hline Dosis mantenimiento TV (mg/día) & 7.5 & 7.5 & 7.5 & 0 & 2.95 & 1.61 & 7.5 & 0 & 15 & 0 & 7.5 & 0 \\
\hline Duración del tratamiento (días) & 770 & 500 & 92.5 & 79 & 596.5 & 985 & 730 & 0 & 61 & 0 & 277 & 630 \\
\hline
\end{tabular}

$\mathrm{SNa}=$ natremia, $\mathrm{TV}=$ tolvaptan, $\mathrm{SIADH}=$ secreción inadecuada de hormona antidiurética, $\mathrm{Me}=$ mediana, DQ1-Q3 = diferencia entre cuartil 1 y cuartil 3.

Tabla 2. Coste del tratamiento ambulatorio de la hiponatremia con tolvaptan por etiología

\begin{tabular}{|c|c|c|c|c|c|c|}
\hline Variable & $\begin{array}{c}\text { SIADH } \\
\text { farmacológica } \\
n=3(33.3 \%)\end{array}$ & $\begin{array}{c}\text { SIADH } \\
\text { paraneoplásica } \\
n=2(22.2 \%)\end{array}$ & $\begin{array}{c}\text { SIADH } \\
2^{\circ} \text { padecimiento } \\
\text { neurológico } \\
n=2(22.2 \%)\end{array}$ & $\begin{array}{c}\text { SIADH } \\
2^{\circ} \text { padecimiento } \\
\text { respiratorio } \\
n=1(11.1 \%)\end{array}$ & $\begin{array}{c}\text { Hiponatremia } \\
\text { dilucional } \\
\text { (cirrosis) } \\
n=1(11.1 \% \text { ) }\end{array}$ & $\begin{array}{l}\text { Total } \\
n=9 \\
(100)\end{array}$ \\
\hline $\begin{array}{l}\text { Coste-tratamiento, } € \\
\text { (DQ1-Q3) }\end{array}$ & $\begin{array}{l}30766.1 \\
(27912.9)\end{array}$ & $\begin{array}{l}3236.3 \\
(994.9)\end{array}$ & $\begin{array}{c}16880.6 \\
(30759.8)\end{array}$ & $\begin{array}{c}27387.1 \\
(0)\end{array}$ & $\begin{array}{l}8714.5 \\
(0)\end{array}$ & $\begin{array}{l}17930.6 \\
(27033)\end{array}$ \\
\hline Coste total, $€$ & 94540.3 & 6472.5 & 33761.3 & 27387.1 & 8714.5 & 170875.7 \\
\hline $\begin{array}{l}\text { Coste total-día de } \\
\text { tratamiento, € }\end{array}$ & 163.7 & 79.9 & 44.1 & 37.5 & 142.9 & 39.9 \\
\hline $\begin{array}{l}\text { Coste total-D episodios } \\
\text { ingreso, } €\end{array}$ & 10504.5 & $0^{*}$ & 11253.8 & 13693.6 & $0^{*}$ & 21359.5 \\
\hline Coste total-D días ingreso, $€$ & 844.2 & 106.1 & 767.3 & 912.9 & 181.6 & 587.2 \\
\hline Coste total-D urgencias, $€$ & 13505.8 & $0^{*}$ & 33761.3 & 13693.6 & $0^{*}$ & 24410.8 \\
\hline
\end{tabular}

DQ1-Q3 = diferencia entre cuartil 1 y cuartil 3, $\mathrm{D}$ = diferencia entre los periodos bajo tratamiento con tolvaptan y periodos equivalentes previos al inicio del tratamiento,

SIADH = secreción inadecuada de hormona antidiurética. *Sin diferencia en episodios.

beneficios clínicos por la corrección de la natremia, que se reduce a la publicación de casos clínicos. ${ }^{9}$ En nuestros pacientes se acumuló un seguimiento de 3993 días, con una mediana de 277 (666) días y un máximo de seguimiento individual de 1089 días (2.98 años), lo que supone una de las fortalezas del estudio. El uso de TV parece asociarse con disminución significativa en días de ingreso por hiponatremia y, en suma, por cualquier patología, asimismo identificamos descenso en asistencia a urgencias y episodios de ingreso en los pacientes tratados con tolvaptan.

Resulta reseñable que la mayoría de los pacientes mantiene la eunatremia con la utilización de dosis inferiores a las indicadas en la ficha técnica. En la misma línea, el grupo del Hospital Clínico San Carlos $^{10}$ comunicó sus resultados de seguimiento en 16 pacientes con tratamiento ambulatorio con TV durante un mínimo de tres años; encontró reducción en las hospitalizaciones, días de estancia y visitas a urgencias.

Debe tenerse en cuenta que los resultados varían notablemente en función de la etiología de la hiponatremia, por ejemplo, los pacientes oncológicos acuden en más ocasiones al servicio de urgencias una vez tratados, probablemente por el avance de la enfermedad de base. Por otra parte, se trata de un estudio retrospectivo no aleatorizado y el número de pacientes es escaso, lo que constituye la mayor limitación de nuestro estudio.

En el análisis de costes no se han tenido en cuenta los gastos derivados del seguimiento ambulatorio de los 
pacientes tratados (consultas, estudios de laboratorio, traslados, etcétera), ni los relacionados con el ingreso o asistencia en servicio de urgencias, por lo que serían necesarios estudios diseñados específicamente para analizar la coste-efectividad de este tratamiento.

\section{Conclusiones}

El uso a largo plazo de tolvaptan parece seguro y se podría relacionar con descenso en los días de hospitalización por hiponatremia.

\section{Financiación}

Esta investigación no recibió subvención pública o de sectores comerciales.

\section{Conflictos de intereses}

David E. Barajas Galindo y Emilia Gómez Hoyos informan haber recibido honorarios de Otsuka Pharmaceutical Co. Ltd. por otros trabajos no relacionados con el presente informe.

\section{Bibliografía}

1. Spasovski G, Vanholder R, Allolio B, Annane D, Ball S, Bichet D, et al. Clinical practice guideline on diagnosis and treatment of hyponatraemia. Eur J Endocrinol. 2014;170:G1-G47.

2. Peri A, Pirozzi N, Parenti G, Festuccia F, Menè P. Hyponatremia and the syndrome of inappropriate secretion of antidiuretic hormone (SIADH). J Endocrinol Invest. 2010;33:671-682.

3. Holland-Bill L, Christiansen CF, Heide-Jørgensen U, Ulrichsen SP Ring T, Jørgensen JO, et al. Hyponatremia and mortality risk: a Danish cohort study of 279508 acutely hospitalized patients. Eur J Endocrinol. 2015;173:71-81

4. Sajadieh A, Binici Z, Mouridsen M, Nielsen OW, Hansen J, Haugaard SB. Mild hyponatremia carries a poor prognosis in community subjects. Am J Med. 2009;122:679-686.

5. Hoorn E, Rivadeneira F, van Meurs JB, Ziere G, Stricker BH, Hofman A, et al. Mild hyponatremia as a risk factor for fractures: the Rotterdam Study. J Bone Miner Res. 2011;26:1822-1828.

6. Verbalis JG, Goldsmith SR, Greenberg A, Korzelius C, Schrier RW, Sterns $\mathrm{RH}$, et al. Diagnosis, evaluation, and treatment of hyponatremia: expert panel recommendations. Am J Med. 2013;126:S1-S42.

7. European Medicines Agency. Samsca (tolvaptan). An overview of Sams$\mathrm{ca}$ and why it is authorized in the EU. Reino Unido: European Medicines Agency; 2018.

8. Berl T, Quittnat-Pelletier F, Verbalis $\mathrm{J}$ et al. Oral tolvaptan is safe and effective in chronic hyponatremia. J Am Soc Nephrol. 2010;21:705-712.

9. Büttner S, Bachmann J, Geiger H, Obermüller N. Long-term vaptan treatment of idiopathic SIADH in an octogenarian. J Clin Med. 2017;6:28.

10. Cuesta-Hernández $M$, Crespo-Hernández I, Amich-Alemany $P$, Gómez-Hoyos E, Sánchez-Gómez N, Santiago A, et al. Correction of sustained hyponatremia secondary to SIAD by the use of chronic tolvaptan therapy is associated with a reduction in Emergency Room visits, hospital admissions and days of hospitalization over a 3-year period. Endocrine Abstracts. 2017;49:GP179. 